\title{
Bisbenzamidine derivative, pentamidine represses DNA damage response through inhibition of histone $\mathrm{H} 2 \mathrm{~A}$ acetylation
}

\author{
Junya Kobayashi" ${ }^{1 *}$, Akihiro Kato ${ }^{1}$, Yosuke Ota ${ }^{1}$, Reiko Ohba ${ }^{1,2}$, Kenshi Komatsu ${ }^{1 *}$
}

\begin{abstract}
Background: MRE11 is an important nuclease which functions in the end-resection step of homologous recombination (HR) repair of DNA double-strand breaks (DSBs). As MRE11-deficient ATLD cells exhibit hyper radiosensitivity and impaired DSB repair, MRE11 inhibitors could possibly function as potent radio-sensitizers. Therefore, we investigated whether a bisbenzamidine derivative, pentamidine, which can inhibit endoexonuclease activity, might influence DSB-induced damage responses via inhibition of MRE11.

Results: We first clarified that pentamidine inhibited MRE11 nuclease activity and also reduced ATM kinase activity in vitro. Pentamidine increased the radio-sensitivity of HeLa cells, suggesting that this compound could possibly influence DNA damage response factors in vivo. Indeed, we found that pentamidine reduced the accumulation of $\gamma-\mathrm{H} 2 \mathrm{AX}, \mathrm{NBS1}$ and phospho-ATM at the sites of DSBs. Furthermore, pentamidine decreased HR activity in vivo. Pentamidine was found to inhibit the acetylation of histone H2A which could contribute both to inhibition of IRinduced focus formation and HR repair. These results suggest that pentamidine might exert its effects by inhibiting histone acetyltransferases. We found that pentamidine repressed the activity of Tip60 acetyltransferase which is known to acetylate histone H2A and that knockdown of Tip60 by siRNA reduced HR activity.

Conclusion: These results indicate that inhibition of Tip60 as well as hMRE11 nuclease by pentamidine underlies the radiosensitizing effects of this compound making it an excellent sensitizer for radiotherapy or chemotherapy.
\end{abstract}

\section{Background}

DNA double-strand breaks (DSBs) are generated by exposure to ionizing radiation, DNA damaging agents such as bleomycin or neocarzinostatin, or due to the stalling or collapse of DNA replication forks. As unrepaired DSBs induce genome instability and promote apoptosis or tumorigenesis, cells recognize DSBs immediately and activate cell cycle checkpoints and DNA repair mechanisms. Hence, the generation of DSBs by exposure to ionizing radiation (IR) could induce cell death in tumor cells and the inhibition of DSB repair activity in tumors might lead to efficient radiotherapy. The generation of DSBs triggers the relocalization of many DNA damage response (DDR) proteins such as MRE11/NBS1/RAD50, MDC1, 53BP1

\footnotetext{
* Correspondence: jkobayashi@house.rbc.kyoto-u.ac.jp; komatsu@house.rbc. kyoto-u.ac.jp

${ }^{1}$ Department of Genome Repair Dynamics, Radiation Biology Center, Kyoto
} University, Kyoto 606-8501, Japan and BRCA1 to nuclear foci that co-localize with $\gamma$ H2AX [1-5]. H2AX is rapidly phosphorylated at DSB sites and phosphorylated $\mathrm{H} 2 \mathrm{AX}(\gamma-\mathrm{H} 2 \mathrm{AX})$ interacts with NBS1, MDC1 and BRCA1, thereby promoting their accumulation at DSBs $[1,6]$. Hence, H2AX-knockout cells are deficient in the formation of DSB-induced nuclear foci of several DDR proteins such as NBS1 $[2,6,7]$. Furthermore, H2AX-knockout cells are defective in homologous recombination (HR) repair [8]. Both $\mathrm{H} 2 \mathrm{AX}+/-$ and $\mathrm{H} 2 \mathrm{AX}-/$ - mouse thymocytes show an increase in chromosomal aberrations $[9,10]$. These facts indicate that $\gamma-\mathrm{H} 2 \mathrm{AX}$-depedent foci formation could be important for DSB repair, particularly HR repair, and genome stability.

MRE11 nuclease is a key factor in DSB damage response and functions as both a single- and doublestranded DNA endonuclease as well as $3^{\prime}->5$ ' exonuclease $[11,12]$. It has been reported that this nuclease activity is indispensable for homologous recombination, 
both during DSB repair and during meiotic recombination using yeast cell lines lacking functional Mre11 $[13,14]$. Mutations in the hMRE11 gene result in Ataxia Telangiectasia-like disorder (AT-LD) syndrome. Both AT-LD patient cells and ATM-defective Ataxia Telagiectasia patients cells show similar phenotypes such as radio-resistant DNA synthesis, radiation hyper-sensitivity and genome instability [15-17]. hMRE11 forms a complex with NBS1 and hRAD50 and this complex displays DNA binding and tethering activities as well as nuclease activity. This complex has been shown to function in DNA double-strand break repair by HR in mammals [18,19]. Moreover, efficient HR repair requires IRinduced focus formation (recruitment) of the NBS1/ hMRE11/hRAD50 complex at DNA damage sites [20]. Hence, the genomic instability in AT-LD patients could be due to the defect in HR. Therefore, the inhibition of hMRE11 nuclease activity or recruitment of this complex may result in radiosensitization.

The bisbenzamidine derivative, pentamidine, has been one of the most successful agents against eukaryotic parasites and has been used clinically against trypanosomiasis, leishmananiasis, and Pneumocystis carinii for over 70 years [21-23]. Pentamidine enters parasite cells rapidly and appear first in the kinetoplast that contains the mitochondrial DNA of the parasite. With time it is also generally seen in the cell nucleus but significant amounts are not observed in the cytoplasm. Pentamidin is capable of binding to the minor groove of doublestrand DNA but not single-strand DNA and inhibits protein synthesis, DNA synthesis and the activity of endo-exonuclease in Pneumocystis carinii [24]. Further, DNA and protein synthesis in human tumor also decreased by pentamidine treatment [25]. Recently, it was reported that pentamidine also inhibited human endo-exonuclease activity in vitro and induced cell death in several tumor cells efficiently [26]. Although it is unclear as to whether pentamidine might inhibit other nucleases such as hMRE11, the effect of pentamidine on hMRE11 could potentially lead to anti-tumorigenic effects or effective radiotherapy.

In this paper, we first demonstrate the inhibitory effect of pentamidine to hMRE11 nuclease activity in vitro. We also show that pentamidine increases the radio-sensitivity of HeLa cells and represses IR-induced focus formation of $\gamma-\mathrm{H} 2 \mathrm{AX}$ and NBS1. Furthermore, pentamidine reduces the HR activity and acetylation of histone H2A, mediated by Tip60 histone acetytransferase (HAT). Moreover, pentamidine reduces the HAT activity of Tip60 in vitro. We discuss that such these novel inhibitory effects of pentamidine on hMRE11 and Tip60 opens up important therapeutic and radiosensitzing options for cancer therapy.

\section{Results}

Pentamidine inhibits in vitro MRE11 nuclease activity and ATM kinase activity

It has been previously reported that some dicationic diaryfurans such as pentamidine inhibit the endo-exonuclease in Pneumocystus carinii [24]. As human endoexonuclease activity was also reported to be repressed pentamidine in vitro [26], we investigated whether pentamidine was capable of inhibiting hMRE11 nuclease activity in vitro (Fig. 1A). Digestion of the hMRE11 substrate was completed by 90 mins. Importantly, upon addition of pentamidine $(2 \mathrm{mM})$ substrate digestion was almost completely inhibited even after 90 mins. Thus, pentamidine could inhibit hMRE11 nuclease activity in vitro. The MRN complex is known to bind with ATM kinase directly and this interaction is essential for the activation of ATM [27]. Hence, we next examined whether pentamidine could influence ATM kinase activity in vitro (Fig. 1B). Immunoprecipitated ATM from un-irradiated cells exhibited low levels of p53 phosphorylation activity and this was increased 1.73 times upon irradiation. However, the addition of pentamidine (1 or $2 \mathrm{mM}$ ) resulted in an obvious decrease in ATM kinase activity. Thus, pentamidine reduced both hMRE11 nuclease and ATM kinase activities in vitro. As these activities are very important for IR-induced DNA damage responses and subsequent fate of the cells, pentamidine would be expected to increase radio-sensitivity. Therefore, the effect of pentamidine treatment on radiosensitivity was examined in HeLa cells (Fig. 1C). The viability after 5 Gy of irradiation is about 0.2 , but the addition of pentamidine $(0.05 \mathrm{mM})$ decreased the cell viability to approximately 0.08 . Further, the treatment of $0.1 \mathrm{mM}$ pentamidine also increased radio-sensitivity in HeLa cells (Additional file 1, Fig. S1). Moreover, 0.05 $\mathrm{mM}$ of pentamidine treatment induced a few multinucleated cells without irradiation, and irradiation increased the number of multinucleated cells (Fig. 1D). Multinucleation upon pentamidine treatment might be a consequence of the cells proceeding into mitosis without appropriate DSB repair or cell cycle checkpoint implementation resulting in mitotic catastrophe.

\section{Pentamidine represses the formation of DNA-damage responsive foci upon irradiation}

Fig. 1 indicated that very low concentration $(0.05 \mathrm{mM})$ of pentamidine influenced the radio-sensitivity and multi-nuclear formation in vivo, although more than 0.5 $\mathrm{mM}$ of pentamidine is required for inhibition to hMRE11 activity or ATM kinase activity in vitro. As hMRE11/ATM are some of the earliest responders to DSBs and trigger many aspects of DDR, pentamidine may influence the function of other factors in DNA 


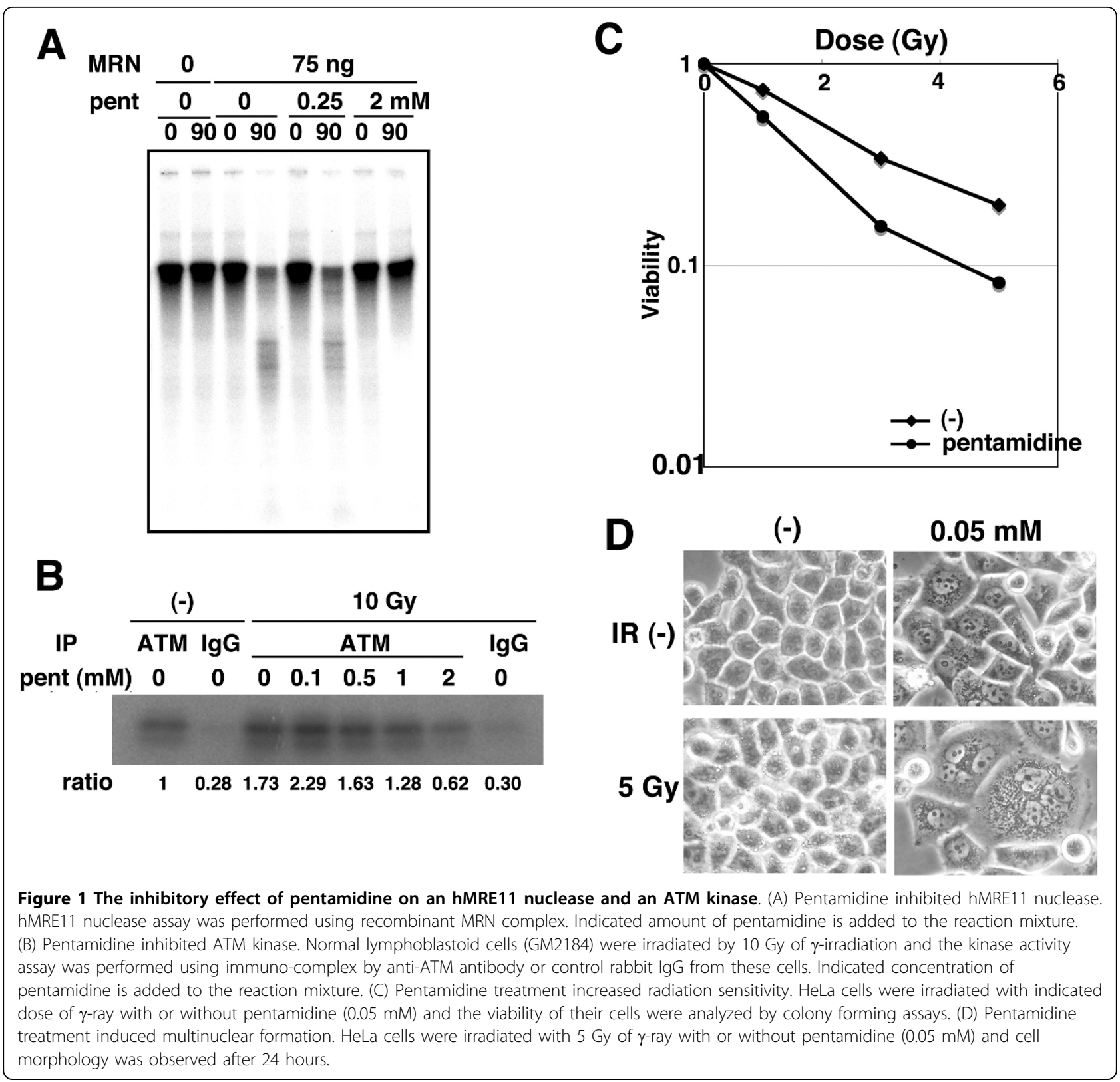

damage response. The accumulation of several DDR proteins into "foci" following IR is early and important event in DNA damage-induced cellular response, particularly $\gamma$-H2AX foci, which are formed before most DNA damage responses. Therefore, we investigated the effect of pentamidine on $\gamma$-H2AX focus formation following IR (Fig. 2AB). Without pentamidine treatment, most cells exhibited $\gamma$-H2AX focus formation after an exposure to 5 Gy of $\gamma$-ray. Pre-treatment with pentamidine (more than $0.5 \mathrm{mM}$ ) resulted in a striking reduction in focus formation. Previously, we reported that the IR-induced focus formation of NBS1, which forms a complex with hMRE11 and hRAD50, is dependent on $\gamma$ -
H2AX [1]; therefore, we verified the inhibitory effect of pentamidine on the formation of NBS1 foci following irradiation (Fig. $2 \mathrm{C}$ and Additional file 1, Fig. S2 and S3). Similarly to $\gamma$-H2AX foci, IR-induced NBS1 focus formation was decreased by pre-treatment of pentamidine. Pentamidine also repressed focus formation by hMRE11, MDC1 and phospho-ATM, whose accumulation at DSBs is dependent on $\gamma-\mathrm{H} 2 \mathrm{AX}$ or NBS1 (Fig. 2C). Recently, Chromatin-immunoprecipitation (ChIP) has been effectively used to clarify the recruitment (accumulation) of DNA damage-related factors to DSB sites [28]. Usually, the recruitment of factors that accumulated as IR-induced foci, is also detectable by ChIP 

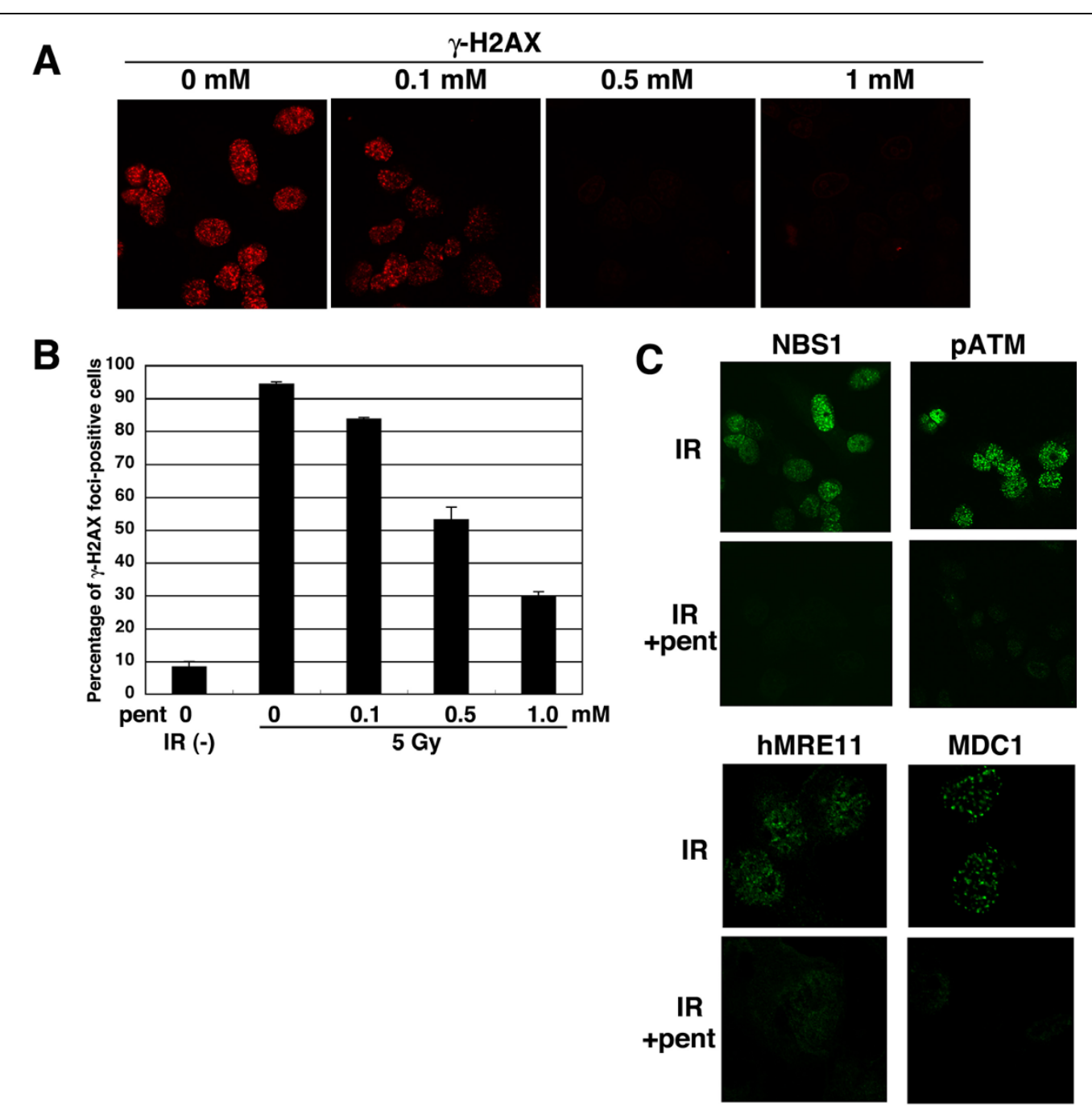

Figure 2 The effect of pentamidine on IR-induced cellular response. (A) Pentamidine repressed $\boldsymbol{\gamma}$-H2AX foci formation. MRC5SV cells were irradiated with 5 Gy of $\gamma$-ray with or without pre-treatment of pentamidine (indicated concentrations, 30 minutes). After 30 minutes, their cells were fixed and immuno-staining was performed using anti- $\gamma-\mathrm{H} 2 \mathrm{AX}$. Percentage of $\gamma$ - $\mathrm{H} 2 \mathrm{AX}$ foci-positive cell was shown in (B). (C) Pentamidine repressed focus formation of DNA damage-related factors. MRC5SV cells were irradiated with 5 Gy of $\gamma$-ray with or without pre-treatment of pentamidine (0.5 mM, 30 minutes). After 30 minutes, their cells were fixed and immuno-staining was performed using anti-NBS1, anti-phosphoATM, anti-hMRE11 and anti-MDC1 antibodies.

assay. When we examined the recruitment of $\gamma-\mathrm{H} 2 \mathrm{AX}$ or NBS1 near DSB sites by ChIP assay, these recruitments were detected without pentamidine treatment (Additional file 1, Fig. S4). However, pre-treatment with pentamidine repressed these recruitments $(\gamma-\mathrm{H} 2 \mathrm{AX}$ : 0 and $4 \mathrm{~kb}$; NBS1: $0 \mathrm{~kb}$ ), which is consistent with the results of focus formation in Fig. 2ABC. However, the accumulation of $\gamma-\mathrm{H} 2 \mathrm{AX}$ at $1 \mathrm{~kb}$ distance was not repressed by pentamidine. As the formation of $\gamma-\mathrm{H} 2 \mathrm{AX}$ is composed by two steps; ATM/NBS1-dependent primary phosphorylation and subsequent MDC1-dependent accumulation [7]. Hence, inhibitory effect of pentamidine might be different between two steps. As Fig. 1B showed that pentamidine could inhibit ATM kinase activity and Fig. 2AB showed that pentamidine reduced ATM-dependent $\gamma$-H2AX focus formation, we investigated whether pentamidine could repress ATM-dependent DDR phosphorylation events (Fig. 3A). Irradiation with $\gamma$-rays (5 Gy) induced phosphorylation of SMC1, Chk2, and p53, for which ATM is the responsible kinase. However, $0.5 \mathrm{mM}$ of pentamidine treatment did not reduce these phosphorylation. Moreover, the treatment of $1 \mathrm{mM}$ of pentamidine, which can diminish almost $\gamma$-H2AX focus formation, did not influence these phosphorylation in both MRC5SV and HeLa cells (Additional file 1, Fig. S5AB). However, $2 \mathrm{mM}$ of pentamidine 


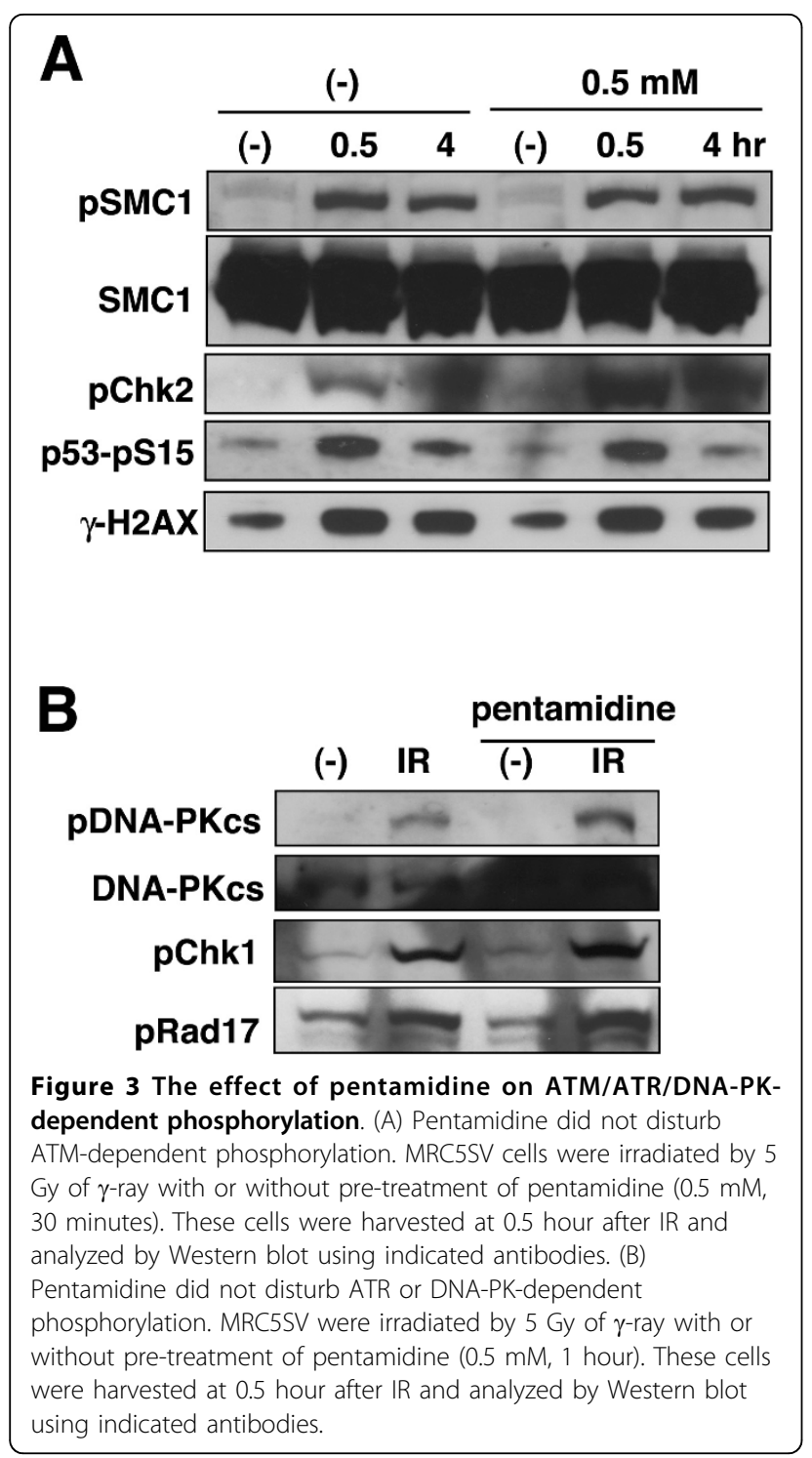

treatment reduced auto-phosphorylation of ATM and SMC1 phosphorylation (Additional file 1, Fig. S5C), which is consistent with the inhibitory effect on ATM kinase activity in vitro (Fig. 1B). Moreover, low concentration of pentamidine also showed no effect on DNAPK or ATR-dependent phosphorylations (Fig. 3B). Taken together, low concentration of pentamidine might repress IR-induced foci formation and accumulation of proteins such as $\gamma-\mathrm{H} 2 \mathrm{AX}$ independently of inhibition to ATM kinase in vivo.

\section{Pentamidine represses both homologous recombination repair and histone acetylation}

Various DNA damage-related proteins form nuclear foci at DSB sites, and many of them, such as the MRN complex, $\gamma$-H2AX, BRCA1, Rad51, are known to function in homologous recombination repair [8,20,29]. As Fig. 2 indicated that pentamidine reduced their focus formation, we next examined whether pentamidine repressed HR repair. When we used the DR-GFP system developed by the Jasin laboratory [30] to estimate HR activity in MRC5SV cells, the generation of DSB by an expression of I-SceI restrection enzyme induced approximate $28 \%$ of GFP-positive cells (Fig. 4A). Pre-treatment with pentamidine reduced GFP-positive cells to $16 \%$ after ISceI introduction. In the case of HeLa cells, similar effect of pentamidine was observed (Fig. 4B). We also estimated the effect of pentamidine on NHEJ repair, but the frequency of GFP-positive cells via NHEJ pathway was unchanged with or without pentamidine treatment (Fig. 4C). These results suggest that prentamidine could repress HR repair activity. Recently, the importance of histone modification, particularly, its acetylation in HR repair has been reported [31]. Using the DR-GFP assay we found that the histone acetytransferase (HAT)-specific inhibitors anacardic acid and curcumin reduced HR activity, but Trichostatin A (histone deacetylase inhibitor) did not influence the HR activity (Additional file 1, Fig. S6), suggesting that histone acetylation is indispensable for HR. Surprisingly, pentamidine reduced the acetylation of histone $\mathrm{H} 2 \mathrm{~A}$ at Lys5 dramatically, and the acetylation of $\mathrm{H} 2 \mathrm{~A}$ at Lys9 was also decreased, although $\gamma$-irradiation did not increase the acetylation of these histones (Fig. 5A). As this acetylation was expected to have an important role in DNA damage responses, we generated exogenous FLAG-H2AX (WT or K5/9R mutants)-expressing MRC5SV cells. K5/9R-H2A-expressing cells exhibited a decrease in NBS1-focus formation following IR (Fig. 5BC). Moreover, the HR activity in K5/9R-H2A-expressing cells was much less than that in WT-H2A-expressing cells (Fig. 5D). Taken together, the acetylation of H2A at Lys5 and Lys9 appears to play an important role in $\mathrm{HR}$ repair and pentamidine might influence the IR-induced focus formation and HR repair through a repression of histone $\mathrm{H} 2 \mathrm{~A}$ acetylation.

\section{Pentamidine inhibits Histone acetyltransferase, Tip60}

Fig. 5 indicates the possibility that pentamidine might inhibit the acetylation of histone $\mathrm{H} 2 \mathrm{~A}$ in addition to inhibiting the activities of hMRE11 and ATM (Fig. $1 \mathrm{AB})$. Hence, the decrease in histone $\mathrm{H} 2 \mathrm{~A}$ acetylation might be an indirect effect of pentamidine through ATM or hMRE11 activity. However, ATM inhibitors did not influence the acetylation of $\mathrm{H} 2 \mathrm{~A}$ with or without irradiation (Additional file 1, Fig. S7A), although it reduced SMC1 phosphorylation. hMRE11-deficient ATLD cells showed normal levels of acetylation (Additional file 1, Fig. S7B), suggesting that hMRE11 nuclease activity is dispensable for an acetylation of histone H2A. 


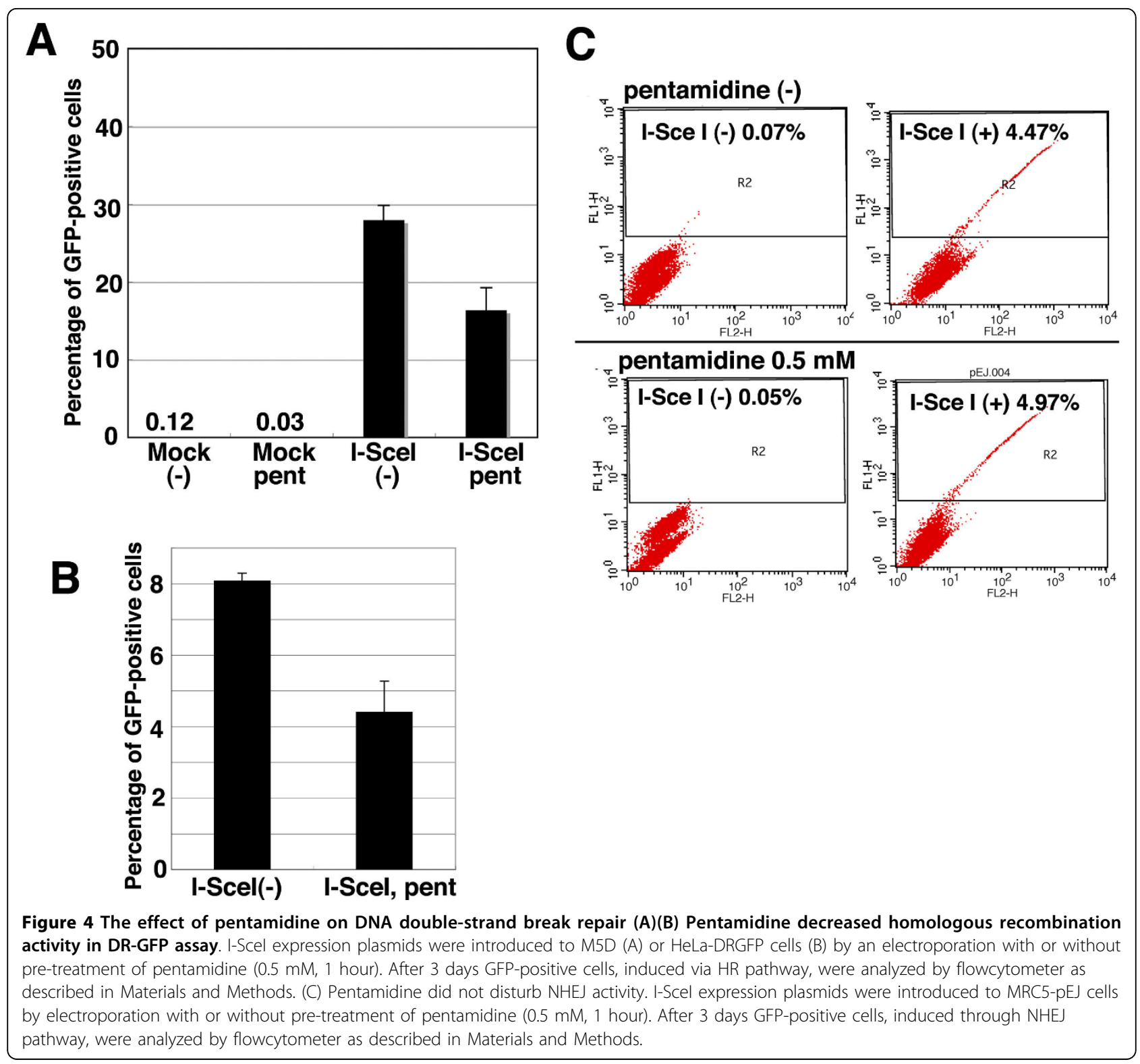

Tip60 histone acetyltransferase is reported to be responsible for histone acetylation and can acetylate histone $\mathrm{H} 2 \mathrm{~A}$ in vitro [32]. Further, several lines of evidence suggests that Tip60 might function in DSB damage response [31,33]. Therefore, we examined whether the repression of Tip60 by siRNA result in an effect that is similar to pentamidine treatment. Tip60-knock-down cells showed clear reduction of $\mathrm{H} 2 \mathrm{~A}$ acetylation at Lys 5 and Lys9, but did not influence the acetylation of $\mathrm{H} 3$, $\mathrm{H} 2 \mathrm{~B}$, and H4 (Fig. 6A). Moreover, the repression of Tip60 also reduced HR activity in DR-GFP system (Fig. 6B). These results suggest that Tip60 is a crucial acetyltransferase for histone $\mathrm{H} 2 \mathrm{~A}$ and plays a role in $\mathrm{HR}$ repair through the acetylation oh histone $\mathrm{H} 2 \mathrm{~A}$. We next investigated if pentamidine directly inhibit Tip60 activity in vitro (Fig. 6C). Immunoprecipitated Tip60 could acetylate recombinant $\mathrm{H} 2 \mathrm{~A}$ in vitro and 10 Gy of irradiation increased this activity. However, the addition of pentamidine abolished the induction of Tip60 activity. Tip60 is reported to acetylate p53 at Lys120 as well as hisotone $\mathrm{H} 2 \mathrm{~A}$ at Lys5 and Lys9 [34]. Pentamidine treatment also reduced Tip60-dependent acetylation of p53 in MRC5SV cells (Fig. 6D), suggesting that pentamidine could inhibit Tip60 acetyltransferase acitivity in vivo. Taken together, pentamidine could repress histone acetylation through direct inhibition of Tip60 histone acetyltransferase, and this repression might leads to a decrease in HR repair activity and cell viability after irradiation. 

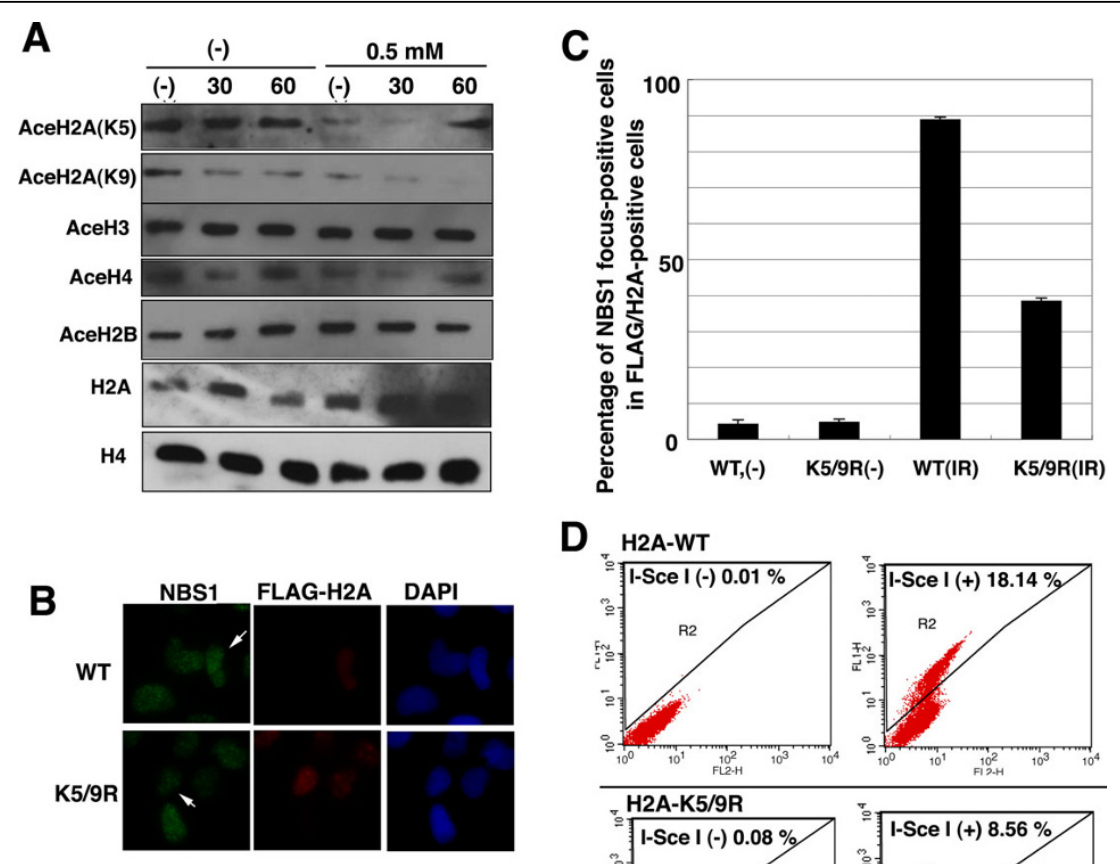

D. H2A-WT

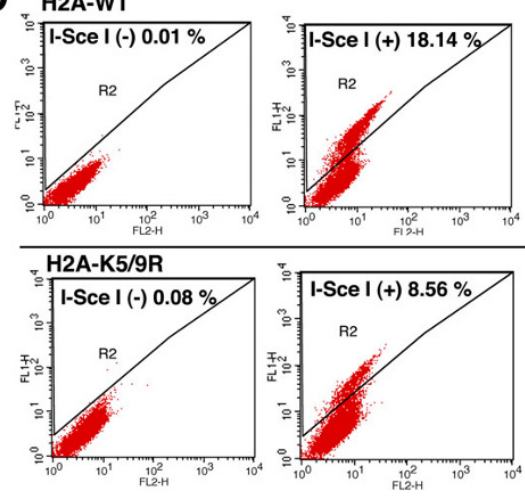

Figure 5 Pentamidine might affect DNA damage response through acetylation of histone H2A. (A) The effect of pentamidine on histone acetylation. MRC5SV cells were irradiated with $5 \mathrm{~Gy}$ of $\gamma$-ray with or without pre-treatment of pentamidine $(0.5 \mathrm{mM}, 30$ minutes). These cells were harvested at indicated times after IR and analyzed by Western blot using indicated antibodies. (B) Acetylation of histone $\mathrm{H} 2 \mathrm{~A}$ is required for IR-induced foci formation. H2A-WT or H2A-K5/9R-expressing cells were generated as described in Materials and Methods. These cells were irradiated with 5 Gy of $\gamma$-ray. After 30 minutes, their cells were fixed and immuno-staining was performed using anti-NBS1 and anti-FLAG antibodies. Percentage of NBS1 foci-positive cells was shown in (C). (D) HR activity in H2A (K5/9R)-expressing cells. I-Scel expression plasmids were introduced into H2A-WT or H2A-K5/9R- expressing cells by electroporation. After 2 days GFP-positive cells, induced through HR pathway, were analyzed by flowcytometer.

\section{Discussion}

We identified here that pentamidine is a novel inhibitor of hMRE11 nuclease (Fig. 1A). Pentamidine also repressed ATM kinase activity in vitro (Fig. 1B). Low concentrations of pentamidine enhanced radio-sensitivity of HeLa cells (Fig. 1C), suggesting that pentamidine might influence other factors in DNA damage response. Pentamidine reduced IR-induced focus formation of DDR proteins such as $\gamma-\mathrm{H} 2 \mathrm{AX}$ and NBS1, although a remarkable effect on ATM-dependent phosphorylations was not observed (Fig. 2 and 3). Pentamidine also repressed HR activity but not NHEJ (Fig 4). Furthemore, pentamidine reduced acetylation of histone H2A (Fig. $5 \mathrm{~A})$, which is suggested to influence IR-induced focus formation and HR repair. H2A mutated at acetylation sites decreased both IR-induced focus formation of NBS1 and HR activity (Fig. 5BCD). Knockdown of Tip60, which is known as a histone acetyl transferase, resulted in effects on $\mathrm{H} 2 \mathrm{~A}$ acetylation and HR activity similarly to that seen upon pentamidine treatment (Fig.
6AB). Moreover, pentamidine reduced HAT activity of Tip60 in vitro and Tip60-dependent acetylation of p53 in vivo (Fig. $6 \mathrm{CD}$ ), suggesting that the inhibitory effects of pentamidine on DDR may be mediated, at least in part, by inhibitin of Tip60. However, the effects of Tip60-knockdown were less pronounced than those by pentamidine treatment (Fig. 6B). Taken together, pentamidine might influence IR-induced DNA damage response through the inhibitory effect on not only Tip60, but also hMRE11 and ATM.

Pentamidine is known to be as one of the most effective agents against Pneumocystis carinii. Pentamidin is capable of binding to the minor groove of double-strand DNA and inhibits protein synthesis, DNA synthesis and the activity of endo-exonuclease in Pneumocystis carinii [24]. Pentamidine also repressed Saccharomyces cerevisiae RNC1/TRM2 endo-exonuclease, which displayed 5'$>3$ ' exonuclease activity on double-strand DNA and endonuclease activity on single-strand DNA in vitro [35]. Recently, it was reported that pentamidine also 


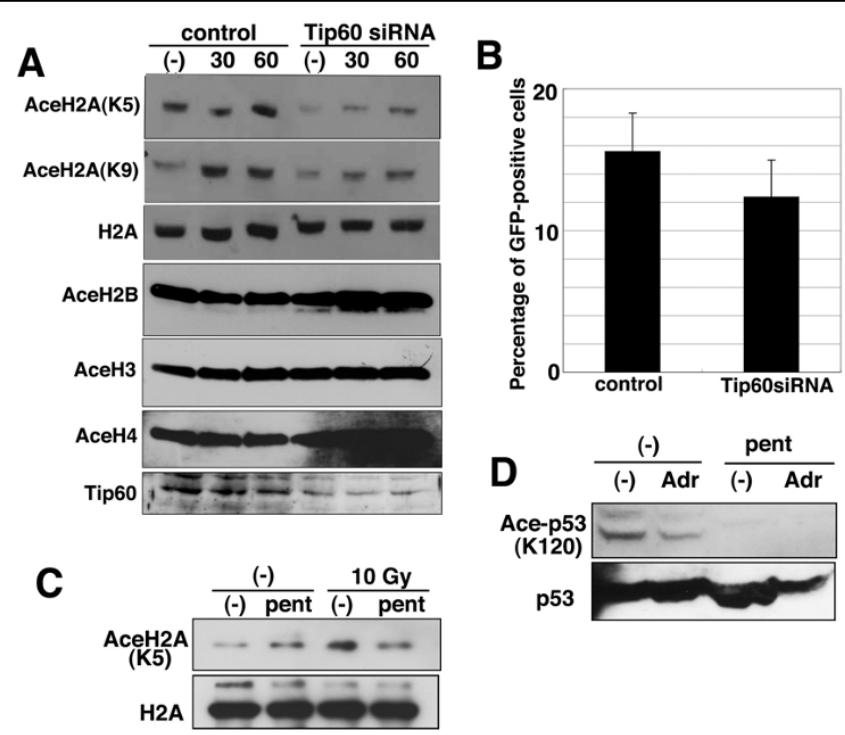

Figure 6 Tip60-knockdown also repressed both HR activity and acetylation of histone H2A. (A) Tip60-knockdown reduced acetylation of histone H2A. MRC5SV cells were transfected by Tip60 siRNA. After 2 days, these cells were irradiated by 5 Gy of $\boldsymbol{\gamma}$-ray and were harvested at indicated times after IR and analyzed by Western blot using indicated antibodies. (B) HR activity in Tip60-knockdowned cells. M5D cells were transfected by Tip60 siRNA. After 2 days, I-Scel expression plasmids were introduced by electroporation. After 3 days, GFP-positive cells were analyzed by flowcytometer. (C) The effect of pentamidine on HAT activity of Tip60. Whole cell extract was prepared from irradiated or unirradiated HeLa cells. And then, Tip60-dependent HAT activity was measured with or without pentamidine (5 $\mu \mathrm{M})$ as described in Material and Methods. (D) Pentamidine repressed Tip60-dependent acetylation of p53. MRC5SV cells were added by 0.5 mM adriamycin with or without pretreatment of pentamidine $(0.5 \mathrm{mM}, 30$ minutes). These cells were harvested after 4 hours and analyzed by Western blot using anti-acetylated p53 antibody.

inhibited human endo-exonuclease activity in vitro [26]. These observations suggest that pentamidine might show an inhibitory effect on other nuclease such as hMRE11 in mammalian cells. Indeed, our results demonstrate that addition of $2 \mathrm{mM}$ pentamidine abolished hMRE11 nucelase activity in vitro (Fig. 1A), suggesting that pentamidine could be used as an effective inhibitor in human cells. We also showed that pentamidine reduced ATM kinase acitivity. As ATM requires MRN complex for optimal activation [27], the effect of pentamidine on ATM kinase activity might occur via repression of hMRE11 nuclease by pentamidine. Future experiments will be aimed at clarifying whether pentamidine reduces ATM kinase activity directly.

Recently, several reports suggest a tight relationship between histone acetylation and DNA damage response. Tamburini and Tyler showed that acetylation of histone $\mathrm{H} 3$ and $\mathrm{H} 4$ increased at $\mathrm{HO}$ endonuclease-restricted DSB sites in yeasts by ChIP assay and also showed that the histone acetyltransferases Gcn5 and Esa1 were recruited at these DSB sites [36]. Downs and his colleagues also reported that Arp4, a subunit of NuA4 HAT complex interacts with phosphorylated H2A directly and Arp4 was recruited to HO-related DSB sites [37]. Further, human homologous of NuA4 HAT, Tip60 interact with hitstone $\mathrm{H} 2 \mathrm{AX}$ and could acetylated it, and also showed that acetylation of histone H2AX increased in response to DSB damage [33]. Furthermore, the strains of $\mathrm{S}$. pombe or $\mathrm{S}$. cerevisieae expressing mutated $\mathrm{H} 3$ or $\mathrm{H} 4$ at acetylation sites or HAT-deficient yeasts increased the sensitivity to DNA damaging agents in yeast and dominant-negative Tip60-expressing HeLa cells showed the delay of DSB repair following IR by comet assay $[32,36]$. These reports suggest that acetylation of histon is important for DNA damage response and, perhaps, DNA repair. This is consistent with our results showing that the $\mathrm{H} 2 \mathrm{~A}-\mathrm{K} 5 / 9 \mathrm{R}$ (acetylation site mutant)-expressing cells showed a decrease in HR repair activity (Fig. 5D) and Tip60-knockdown cells also reduced the HR activity (Fig. 6B). We also indicated that pentamidine treatment and the $\mathrm{H} 2 \mathrm{~A}$ (K5/9R)-expressing cells repressed both focus formation and HR (Figs $2 \mathrm{ABC}, 4 \mathrm{AB}$ and $5 \mathrm{BCD}$ ), suggesting that focus formation of DNA damage-related factors such as NBS1 and $\gamma$ H2AX contributes to HR repair pathway through histone acetylation. In fact, a partner protein of Tip60, TRAPP-knockout mouse cells showed a decrease in accumulation of histone acetylation at DSB sites, IRinduced focus formation such as BRCA1 or Rad51 and HR activity [31]. Moreover, we reported that the defect of MRE11 focus formation in mutated NBS1-expressing cells leads to reduction of HR activity [20]. Thus, focus formation of DNA damage response factors could be closely related with homologous recombination repair. 
Moreover, pentamidine might influence HR repair through repression of histone acetylation, related with IR-induced focus formation.

So far, it was reported that the bisbenzamidine derivative, pentamidine showed the growth repression against several tumor cells such as MCF7 and HeLa cells via repression of endo-exonuclease activity [26]. The paper also showed that normal human diploid fibroblats are not sensitive to pentamidine and pentamidine displayed an inhibitory effect on tumor growth in mouse model. These facts suggest that pentamidine might be an effective anti-tumor reagent. Moreover, pentamidine has a synergistic inhibitory effect to tumor growth with mitomycin $\mathrm{C}$ or other anti-growth reagent, suggesting that pentamidine may show a synergistic action with ionizing radiation $[38,39]$. In fact, Fig. $1 \mathrm{C}$ shows the expected effect with irradiation of $\gamma$-ray, and hence lower concentration $(0.05 \mathrm{mM})$ of pentamidine might be an effective sensitizer for radiotherapy. However, much higher concentration of pentamidine was required for inhibitory effects on Mre11 nuclease and ATM kinase activity in vitro (Fig. $1 \mathrm{AB}$ ), while middle range concentration of pentamidine reduced Tip60 activity in vitro and in vivo (Fig. 6CD). These results suggest that the effect of pentamidine (low concentration) as a radiation sensitizer could be due to an inhibitory effect on Tip60. In order to use pentamidine or other deamidine analogues with radiotherapy or chemotherapy, we need to clarify all the targets of these reagents in DNA damage response and the underlying mechanisms in detail.

\section{Conclusion}

In conclusion we have found the inhibitory effect of pentamidine on Tip60 acetyltransferase and ATM kinase. This effect caused a repression of DDR factors' focus formation and an enhancement of radio-sensitivity particularly via a reduction of histone $\mathrm{H} 2 \mathrm{~A}$ acetylation. We also found that both the acetylation of histone $\mathrm{H} 2 \mathrm{~A}$ and the role Tip60 are important for IR-induced focus formation and homologous recombination repair. Therefore, the further research of pentamidine could find an excellent sensitizer for radiotherapy or chemotherapy.

\section{Materials and methods Pentamidine}

Pentamidine (pentamidine isethionate; Chugai Co., Japan) is dissolved in sterile water and stored at $4{ }^{\circ} \mathrm{C}$.

\section{Cell lines}

MRC5SV cells were SV40-transformed MRC5 human fibroblasts $[7,40]$. NBS fibroblast cell line, GM07166VA7, was established by SV40-transformation of GM07166 cells, which were provided from NIGMS
Cell Repository [40]. AT-LD fibroblast cell line, ATLD2SV was established by SV40-transformation of ATLD2 primary fibroblasts, which were supplied by Dr Y. Shiloh [41]. Normal lymphoblastoid cell line, GM2184 was also obtained from NIGMS Cell Repository. H2A (WT) or H2A (K5/9R)-expressing M5D cells were generated by a transfection of pCMV-Tag2 plasmid (Stratagene) inserted with human H2A cDNA. After G418 selection, their expression in isolated cells were confirmed by Western blot analysis.

\section{Antibodies}

Phospho-ATM (S1981) rabbit polyclonal antibody (Epitomics Inc.), phospho-SMC1 (S966) rabbit polyclonal and SMC1 rabbit polyclonal, MDC1 rabbit polyclonal antibodies (Bethyl Laboratories Inc.), phospho-p53 (S15) mouse monoclonal, phospho-Chk2 (T68) rabbit polyclonal antibodies (Cell Signaling Technology), hMre11 rabbit polyclonal and Nbs1 rabbit polyclonal antibodies (Novus Biologicals), and Chk2 rabbit polyclonal and anti Tip60 rabbit polyclonal antibodies (Santa Cruz Biotechnology), and $\gamma$ H2AX mouse monoclonal acetyl H2A (K5) rabbit polyclonal, acetyl H2A (K9) rabbit polyclonal, acetyl H2B rabbit polyclonal, acetyl H3 rabbit polyclonal, acetyl $\mathrm{H} 4$ rabbit polyclonal, histone $\mathrm{H} 2 \mathrm{~A}$ rabbit polyclonal and histone $\mathrm{H} 4$ rabbit polyclonal antibodies (Millipore Co.), and p53-acetyl-K120 mouse monoclonal (BioAcademia) were used for Western blot analysis or immuno-staining.

\section{SiRNA knock-down experiments}

Sub-confluent cells, seeded to culture dishes the day before, were transfected by Tip 60 (Be-Bridge International Inc.) or negative (Be-Bridge International Inc.) siRNA using lipofectamine 2000 (Invitrogen Life Technology). After 2 days, these cells were re-seeded in proper culture dishes. Next day, these cells were used for Western blot or DR-GFP analysis.

\section{Western Blot analysis}

Western blot analysis was carried out as described previously [1]. Target proteins were detected with primary antibodies, mentioned above, and HRP-conjugated antirabbit IgG or anti-mouse IgG antibodies (GE Helthcare), and then visualized with an ECL plus chemiluminescence system (GE Helthcare).

\section{Immunofluorescent staining}

Immunofluorescent staining was carried out as described previously [1]. Alexa-488-conjugated anti-rabbit IgG (Molecular Probes) or Alexa-594-conjugated anti-mouse IgG (Molecular Probes) were used for visualization of foci of target protein. 


\section{ATM kinase assay}

ATM kinase assay was carried out as described previously [7]. ATM was immunoprecipitated with an antiATM antibody (Calbiochem). Phosphorylation of p53 (substrate) by immunoprecipitated ATM in vitro in the presence of $\left[\gamma_{-}{ }^{32} \mathrm{P}\right]$-ATP was detected by BAS2000 (FUJI Film Co.).

\section{MRE11 nuclease assay}

MRE11 nuclease assay was performed as previously reported [11,12]. MRN complex proteins for this assay are purified as previously reported [12]. MRE11 nuclease reactions (containing $25 \mathrm{mM}$ MOPS [pH 7.0], $50 \mathrm{mM}$ $\mathrm{NaCl}, 2 \mathrm{mM}$ DTT, $1 \mathrm{mM} \mathrm{MnCl}_{2}, 0.1$ pmole of DNA substrate labeled by $\left[\gamma_{-}{ }^{32} \mathrm{P}\right]-\mathrm{ATP}$ and $150 \mathrm{ng}$ of MRN complexes), were performed in a volume of $10 \mu \mathrm{l}$, and were incubated at $37^{\circ} \mathrm{C}$ for $30 \mathrm{~min}$. Reactions were stopped by the addition of EDTA and SDS to final concentrations of $10 \mathrm{mM}$ and $0.2 \%$, respectively. Each reaction was dried down, resuspended in $5 \mu \mathrm{l}$ of formamide loading buffer, and then loaded onto a sequencing gel containing $15 \%-22.5 \%$ acrylamide and $7 \mathrm{M}$. After the run, each gel was analyzed with BASS2000.

\section{Cell survival assay}

For radiation sensitivity assays, the cells were trypsinized and irradiated with 1,3 , or 5 Gy of ${ }^{60} \mathrm{Co} \gamma$-ray at a dose rate of $1.1 \mathrm{~Gy} / \mathrm{min}$. Immediately after irradiation, cells were plated into $100-\mathrm{mm}$ dishes at such a cell density that 30-40 cells would survive, and incubated for 14 days. The dishes were fixed with ethanol, stained with $4 \%$ Giemsa, and the number of colonies were counted. Surviving fractions were calculated by comparing the number of colonies formed by irradiated cells with the number of colonies formed by non-irradiated control cells. Each result represents an average value from 3 experiments.

\section{Homologous Recombination assay (DR-GFP) assay}

DR-GFP was performed as previously reported [20,30]. To measure the repair of an I-SceI-generated DSBs, 50 $\mu \mathrm{g}$ of the I-SceI expression vector (pCBASce) was introduced to 1000000 M5D cells, by electroporation (GenePulser; BIO-RAD). To determine the amount of HR repair, the percentage of cells, that were GFP-positive, was quantified by flow cytometry 3 days after electroporation with FACScalibur (Becton Dickinson).

\section{NHEJ assay}

NHEJ was performed as previously reported [42]. To measure the repair of an I-SceI-generated DSBs via NHEJ pathway, $50 \mathrm{mg}$ of the I-SceI expression vector pCBASce was introduced to 1000000 MRC5SV-pEJ cells, by electroporation (BIO-RAD). To determine the amount of NHEJ repair, the percentage of GFP-positive cells was quantitated with flow cytometric analysis at 3 days after electroporation by FACScalibur (Becton Dickinson).

\section{In vitro histone acetylation assay by Tip60}

DNA damage-treated or untreated cells were lysed in IP buffer $(150 \mathrm{mM}$ sodium chloride, $10 \mathrm{mM}$ Tris $/ \mathrm{HCl}$ [pH7.4] and $0.5 \%$ NP40) containing a protease inhibitor cocktail (Roch) and sodium orthovanadate for $15 \mathrm{~min}$. Lysates were centrifuged at $20,000 \times \mathrm{g}$ for $30 \mathrm{~min}$ to remove un-solubulized debris. Lysates were pre-cleared with protein A-Sepharose beads (GE healthcare), and immunoprecipitation was performed by incubating the samples with anti-Tip60 antibody (Santa Cruz) at $4{ }^{\circ} \mathrm{C}$ for 1 hour. After washing by IP buffer, these immunoprecipitants were used for in vitro histone acetylation assay. Reaction of Tip60-dependent histone acetylation (50 mM Tris/HCl [pH 8.0], 10\% grycerol, $0.1 \mathrm{mM}$ EDTA, $1 \mathrm{mM}$ DTT, $1 \mathrm{mM}$ PMSF, $10 \mathrm{mM}$ sodium Butyrate, $20 \mathrm{mM}$ Acetyl CoA, $10 \mu \mathrm{g}$ of histone $\mathrm{H} 2 \mathrm{~A}$ ) was performed at $37^{\circ} \mathrm{C}$ for 30 minutes and the reaction was stopped by addition of SDS-PAGE loading buffer. The actylated histone $\mathrm{H} 2 \mathrm{~A}$ in the reaction was detected by Western blot analysis using anti-acetyl H2A (K9) rabbit polyclonal antibody.

\section{Acknowledgements}

We thank Dr Sandeep Burma for the critical reading of the article. We also thank Dr Tanya T Paull for providing bacmid PTP40 and PTP43; Dr David J Chen and Dr Benjamin Chen for providing anti-phospho-DNA-PKcs antibody; Dr Jochen Dahm-Daphi for providing pEJ plasmid. This work was supported by Grants from Ministry of Education, Culture, Sports, Science and Technology of Japan (JK, KK).

\section{Author details}

${ }^{1}$ Department of Genome Repair Dynamics, Radiation Biology Center, Kyoto University, Kyoto 606-8501, Japan. ${ }^{2}$ National Institute of Genetics, Yata 1111, Mishima, Sizuoka 411-8540, Japan.

\section{Authors' contributions}

JK designed the experiments, carried out a large part of experiments and drafted the manuscript. AK performed the MRE11 nuclease assay. YO performed the experiments about immunofluorescent staining and western blot in part. RO carried out ChIP analysis. KK supervised the project and commented on the manuscript. All authors read and approved the final manuscript.

\section{Competing interests}

The authors declare that they have no competing intereats.

Received: 5 November 2009

Accepted: 9 February 2010 Published: 9 February 2010

\section{References}

1. Kobayashi J, Tauchi H, Sakamoto S, Nakamura A, Morishima K, Matsuura S, Kobayashi T, Tamai K, Tanimoto K, Komatsu K: NBS1 localizes to gamma$\mathrm{H} 2 \mathrm{AX}$ foci through interaction with the FHA/BRCT domain. Curr Biol 2002, 12:1846-1851.

2. Celeste A, Petersen S, Romanienko PJ, Fernandez-Capetillo O, Chen HT, Sedelnikova OA, Reina-San-Martin B, Coppola V, Meffre E, 
Difilippantonio MJ, Redon C, Pilch DR, Olaru A, Eckhaus M, CameriniOtero RD, Tessarollo L, Livak F, Manova K, Bonner WM, Nussenzweig MC, Nussenzweig A: Genomic instability in mice lacking histone H2AX. Science 2002, 296:922-927.

3. Stucki M, Jackson S: gammaH2AX and MDC1: anchoring the DNAdamage-response machinery to broken chromosomes. DNA Repair 2006, 5:534-543.

4. Kobayashi J, Antoccia A, Tauchi H, Matsuura S, Komatsu K: NBS1 and its functional role in the DNA damage response. DNA Repair 2004, 3:855-861.

5. Paull TT, Rogakou E, Yamazaki V, Kirchgessner CU, Gellert M, Bonner WM: A critical role for histone $\mathrm{H} 2 \mathrm{AX}$ in recruitment of repair factors to nuclear foci after DNA damage. Curr Biol 2000, 10:886-895.

6. Stewart GS, Wang B, Bignell CR, Taylor AM, Elledge SJ: MDC1 is a mediator of the mammalian DNA damage checkpoint. Nature 2003, 421:961-966.

7. Kobayashi J, Tauchi H, Chen B, Burma S, Tashiro S, Matsuura S, Tanimoto K, Chen DJ, Komatsu K: Histone H2AX participates the DNA damageinduced ATM activation through interaction with NBS1. Biochem Biophys Res Commun 2009, 380:752-757.

8. Xie A, Puget N, Shim I, Odate S, Jarzyna I, Bassing CH, Alt FW, Scully R: Control of sister chromatid recombination by histone H2AX. Molecular Cell 2007, 16:1017-1025.

9. Bassing $\mathrm{CH}$, Suh $\mathrm{H}$, Ferguson DO, Chua KF, Manis J, Eckersdorff M, Gleason M, Bronson R, Lee C, Alt FW: Histone H2AX: a dosage-dependent suppressor of oncogenic translocations and tumors. Cell 2003, 114:359-370.

10. Celeste A, Difilippantonio S, Difilippantonio MJ, Fernandez-Capetillo O, Pilch DR, Sedelnikova OA, Eckhaus M, Ried T, Bonner WM, Nussenzweig A: $\mathrm{H} 2 \mathrm{AX}$ haploinsufficiency modifies genomic stability and tumor susceptibility. Cell 2003, 114:371-383.

11. Paull TT, Gellert M: The $3^{\prime}$ to $5^{\prime}$ exonuclease activity of Mre 11 facilitates repair of DNA double-strand breaks. Mol Cell 1998, 1:969-979.

12. Paull TT, Gellert M: Nbs1 potentiates ATP-driven DNA unwinding and endonuclease cleavage by the Mre11/Rad50 complex. Genes Dev 1999, 13:1276-1288.

13. Dolganov GM, Maser R, Novikov A, Tosto L, Chong S, Bressan DA, Petrini JH: Human Rad50 is physically associated with human Mre11: identification of a conserved multiprotein complex implicated in recombinational DNA repair. Mol Cell Biol 1996, 16:4832-4841.

14. Johzuka K, Ogawa H: Interaction of Mre11 and Rad50: two proteins required for DNA repair and meiosis-specific double-strand break formation in Saccharomyces cerevisiae. Genetics 1995, 139:1521-1532.

15. Stewart GS, Maser R, Stankovic T, Bressan DA, Kaplan Ml, Jaspers NG, Raams A, Byrd PJ, Petrini JH, Taylor AM: The DNA double-strand break repair gene hMRE11 is mutated in individuals with an ataxiatelangiectasia-like disorder. Cell 1999, 10:577-587.

16. Pitts SA, Kullar H, Stankovic T, Stewart GS, Last Jl, Bedenham T, Armstrong SJ, Piane M, Chessa L, Taylor AM, Byrd PJ: hMRE11: genomic structure and a null mutation identified in a transcript protected from nonsense-mediated mRNA decay. Hum Mol Genet 2001, 10:1155-1162.

17. Delia D, Piane M, Buscemi G, Savio C, Palmeri S, Lulli P, Carlessi L, Fontanella $E$, Chessa L: MRE11 mutations and impaired ATM-dependent responses in an Italian family with ataxia-telangiectasia-like disorder. Hum Mol Genet 2004, 13:2155-2163.

18. Yamaguchi-Iwai Y, Sonoda E, Sasaki MS, Morrison C, Haraguchi T, Hiraoka Y, Yamashita YM, Yagi T, Takata M, Price C, Kakazu N, Takeda S: Mre11 is essential for the maintenance of chromosomal DNA in vertebrate cells. EMBO J 1999, 18:6619-6629.

19. Tauchi H, Kobayashi J, Morishima K, van Gent DC, Shiraishi T, Verkaik NS, vanHeems D, Ito E, Nakamura A, Sonoda E, Takata M, Takeda S, Matsuura S, Komatsu K: Nbs1 is essential for DNA repair by homologous recombination in higher vertebrate cells. Nature 2002, 420:93-98.

20. Sakamoto S, lijima K, Mochizuki D, Nakamura K, Teshigawara K, Kobayashi J, Matsuura S, Tauchi H, Komatsu K: Homologous recombination repair is regulated by domains at the $\mathrm{N}$ - and C-terminus of NBS1 and is dissociated with ATM functions. Oncogene 2007, 26:6002-6009.

21. Burchmore RJ, Ogbunude P, Enanga B, Barrett MP: Chemotherapy of human African trypanosomiasis. Curr Pharm Des 2002, 8:256-267.

22. Bakshi RP, Shapiro T: DNA topoisomerases as targets for antiprotozoal therapy. Mini Rev Med Chem 2003, 3:597-608.

23. Wilson WD, Tanious F, Mathis A, Tevis D, Hall JE, Boykin DW: Antiparasitic compounds that target DNA. Biochimie 2008, 90:999-1014.
24. Hildebrandt E, Boykin D, Kumar A, Tidwell RR, Dykstra CC: Identification and characterization of an endo/exonuclease in Pneumocystis carinii that is inhibited by dicationic diarylfurans with efficacy against Pneumocystis pneumonia. J Eukaryot Microbiol 1998, 45:112-121.

25. Bornstein RS, Yarbro J: An evaluation of the mechanism of action of pentamidine isethionate. J Surg Oncol 1970, 2:393-398.

26. Chow TY, Alaoui-Jamali M, Yeh C, Yuen L, Griller D: The DNA doublestranded break repair protein endo-exonuclease as a therapeutic target for cancer. Mol Cancer Ther 2004, 3:911-919.

27. Falck J, Coates J, Jackson SP: Conserved modes of recruitment of ATM, ATR and DNA-PKcs to sites of DNA damage. Nature 2005, 434:605-611.

28. Potts PR, Porteus M, Yu H: Human SMC5/6 complex promotes sister chromatid homologous recombination by recruiting the $\mathrm{SMC} 1 / 3$ cohesin complex to double-strand breaks. EMBO J 2006, 25:3377-3388.

29. lijima K, Ohara M, Seki R, Tauchi H: Dancing on damaged chromatin: functions of ATM and the RAD50/MRE11/NBS1 complex in cellular responses to DNA damage. J Radiat Res 2008, 49:451-464.

30. Pierce $A J$, Jasin M: Measuring recombination proficiency in mouse embryonic stem cells. Methods Mol Biol 2005, 291:373-384.

31. Murr R, Loizou J, Yang YG, Cuenin C, Li H, Wang ZQ, Herceg Z: Histone acetylation by Trrap-Tip60 modulates loading of repair proteins and repair of DNA double-strand breaks. Nat Cell Biol 2006, 8:91-99.

32. Ikura T, Ogryzko V, Grigoriev M, Groisman R, Wang J, Horikoshi M, Scully R, Qin J, Nakatani Y: Involvement of the TIP60 histone acetylase complex in DNA repair and apoptosis. Cell 2000, 102:463-473.

33. Ikura T, Tashiro S, Kakino A, Shima H, Jacob N, Amunugama R, Yoder K, Izumi S, Kuraoka I, Tanaka K, Kimura H, Ikura M, Nishikubo S, Ito T, Muto A, Miyagawa K, Takeda S, Fishel R, Igarashi K, Kamiya K: DNA damagedependent acetylation and ubiquitination of $\mathrm{H} 2 \mathrm{AX}$ enhances chromatin dynamics. Mol Cell Biol 2007, 27:7028-7040.

34. Tang Y, Luo J, Zhang W, Gu W: Tip60-dependent acetylation of p53 modulates the decision between cell-cycle arrest and apoptosis. Mol Cell 2006, 24:827-839.

35. Choudhury SA, Asefa B, Webb A, Ramotar D, Chow TY: Functional and genetic analysis of the Saccharomyces cerevisiae RNC1/TRM2: evidences for its involvement in DNA double-strand break repair. Mol Cell Biochem 2007, 300:215-226.

36. Tamburini BA, Tyler J: Localized histone acetylation and deacetylation triggered by the homologous recombination pathway of double-strand DNA repair. Mol Cell Biol 2005, 25:4903-4913.

37. Downs JA, Allard S, Jobin-Robitaille O, Javaheri A, Auger A, Bouchard N, Kron SJ, Jackson SP, Côté J: Binding of chromatin-modifying activities to phosphorylated histone H2A at DNA damage sites. Mol Cell 2004, 16:979-990.

38. Lee MS, Johansen L, Zhang Y, Wilson A, Keegan M, Avery W, Elliott P, Borisy AA, Keith $C T$ : The novel combination of chlorpromazine and pentamidine exerts synergistic antiproliferative effects through dual mitotic action. Cancer Res 2007, 67:11359-11367.

39. Singh G, Dey C: Induction of apoptosis-like cell death by pentamidine and doxorubicin through differential inhibition of topoisomerase II in arsenite-resistant L. donovani. Acta Trop 2007, 103:172-185.

40. Ito A, Tauchi H, Kobayashi J, Morishima K, Nakamura A, Hirokawa Y, Matsuura S, Ito K, Komatsu K: Expression of full-length NBS1protein restores normal radiation responses in cells from Nijmegen breakage syndrome patients. Biochem Biophys Res Commun 1999, 265:716-721.

41. Andegeko Y, Moyal L, Mittelman L, Tsarfaty I, Shiloh Y, Rotman G: Nuclear retention of ATM at sites of DNA double strand breaks. J Biol Chem 2002, 276:38224-38230.

42. Mansour WY, Schumacher S, Rosskopf R, Rhein T, Schmidt-Petersen F, Gatzemeier F, Haag F, Borgmann K, Willers H, Dahm-Daphi J: Hierarchy of nonhomologous end-joining, single-strand annealing and gene conversion at site-directed DNA double-strand breaks. Nucleic Acids Res 2008, 36:4088-4098.

doi:10.1186/1476-4598-9-34

Cite this article as: Kobayashi et al:: Bisbenzamidine derivative, pentamidine represses DNA damage response through inhibition of histone H2A acetylation. Molecular Cancer 2010 9:34. 